Journal of Theoretical and Applied Mechanics, Sofia, Vol.49 No.1 (2019) pp. 83-93 INTERDISCIPLINARY TOPICS

\title{
SURFACE ROUGHNESS ESTIMATION OF DENTAL MATERIALS USING SPECKLE CONTRAST
}

\author{
Simeon Karpuzov ${ }^{1 *}$, Gergo Mitov ${ }^{2}$,Assen Shulev ${ }^{1}$ \\ ${ }^{1}$ Institute of Mechanics, Bulgarian Academy of Sciences, Sofia 1113, Bulgaria \\ ${ }^{2}$ Steiner Landstrae 124 A-3500, Krems-Stein, Austria \\ [Received 17 August 2018. Accepted 18 February 2019] \\ doi: 10.7546/JTAM.49.19.01.08
}

\begin{abstract}
In this paper a simple technique for surface roughness estimation of composite dental material samples widely used in modern stomatology is presented. The proposed method is easily applicable and based on statistical properties of diffusely scattered laser light. The main challenge we addressed was the specific structural and optical properties, as well as the complex geometric shapes of real dental restorations. A relation between surface roughness and speckle contrast, used in the presented method, is verified.
\end{abstract}

KEY WORDS: speckle, roughness, contrast, light scattering.

\section{INTRODUCTION}

There are various reasons that cause the forming of imperfections on an object's surface. The object and polishing tool geometry, material and structure, as well as the polishing mode of operation and standard usage can cause differences in surface roughness. These differences have an effect on a variety of exploitation characteristics, such as wear and compatibility. Preparing a smooth surface for ceramic restorations is considered as an important requirement for their long-term clinical success. Different steps in the production of dental restrations like grinding, polishing and glazing can significantly influence the surface quality. The surface roughness has a distinctive impact on many of the properties of modern dental ceramic restorations. High surface roughness is one of the main factors, associated with excessive wear of the enamel of opposing teeth [1-3]. High surface roughness of restorations is also reported to decrease colour stability [4] and facilitate bacterial attachment [5,6], hence reducing the optical and biological material properties. Finally, high surface roughness has been often described to generally reduce mechanical properties of different types of ceramic materials [7-11]. Literature recommendations for maximum mean surface roughness for monolithic zirconia restorations vary between 40 and $75 \mu \mathrm{m}[12]$.

\footnotetext{
${ }^{*}$ Corresponding author e-mail: skarpuzov@imbm.bas.bg
} 
Throughout the years, numerous roughness measurement techniques have been developed. Mainly, they are separated into two groups - contact and non-contact methods. The first group contains different contact profilometric methods [13] and AFM [14], while the second includes various optical and non-optical techniques reviewed in [15]. Some of the more popular optical methods are white light [16] and laser [17] techniques, while a commonly used non-optical technique is the ultrasound method [18]. The methods in both groups have their advantages and drawbacks so the choice of an appropriate technique must be done according to the examined material.

Nowadays, light scattering from rough surfaces is often used to obtain information about the surface roughness of a specimen $[17,19,20]$. A phenomenon that is often utilized when illuminating a rough surface with a coherent light source such as a laser is the speckle pattern. There are various techniques using different characteristics of the speckle pattern, such as the mean intensity [21], correlation of speckle images, illuminated at different angles [22], average speckle size [23-25] and speckle-pattern illumination [26,27].

Speckle contrast is also a major property of the speckle pattern that can give information about the surface roughness. The relation between surface roughness and speckle contrast has been extensively researched [28-30]. In those works, a strong correlation between speckle image contrast and surface roughness is demonstrated. The method is shown to be simple and reliable. In [29] speckle contrast is defined as

$$
V=\frac{\sigma_{I}}{\langle I\rangle}
$$

where $\sigma_{I}$ is the standard deviation of the intensity of the speckle image, and $\langle I\rangle$ is the mean intensity.

In $[28,29]$ the laser beam used to illuminate the surface is expanded and collimated. This enables roughness evaluation over a large region. In the mentioned works a far-field approximation is used. Surfaces are illuminated normally.

The results from both works show that the speckle image contrast increases with the increase of surface roughness.

\section{Roughness Measurement Technique}

The current work is based on the technique shown in [29] with a few modifications. Firstly, the laser beam is not expanded, as local evaluation is required due to the complex shape of the examined surface. Secondly, the camera is in close proximity to the specimen that allows registering a larger part of the scattered light. Illumination is done at normal incidence with respect to the specimen surface.

Several problems related to the specimens' features have been dealt with: they are a mixture of transparent polymer and opaque zirconia particles and their surface 
is not flat. Their transparency leads to an appearance of speckle patterns from the inside of the specimen, which affects the estimation of the contrast $V$. Illumination and contrast estimation was done only for the outer convex parts of the crowns. These parts contribute the most to enamel wear of opposing teeth and only for them can a correct illumination angle be set-up. Their complex shape introduces a challenge in regards to the set-up of the correct illumination angle. Additionally, it was found that for high-powered laser beams the surfaces could heat up, which also might affect $V$.

The experimental set-up used to record the speckle contrast produced by different specimens is shown in Fig. 1. The source of coherent light is a He-Ne laser. The laser beam illuminates the object at a fixed angle. The specimens are placed on a precise positioning module providing 5 degrees of freedom ( 3 translations and 2 rotations). The illumination angle is controlled by carefully setting the reflected beam on a target, placed opposite of the laser. A CMOS camera captures the intensity of the scattered light. The recorded images are then processed on a computer. The laser beam is not expanded. This enables local estimation of surface roughness.

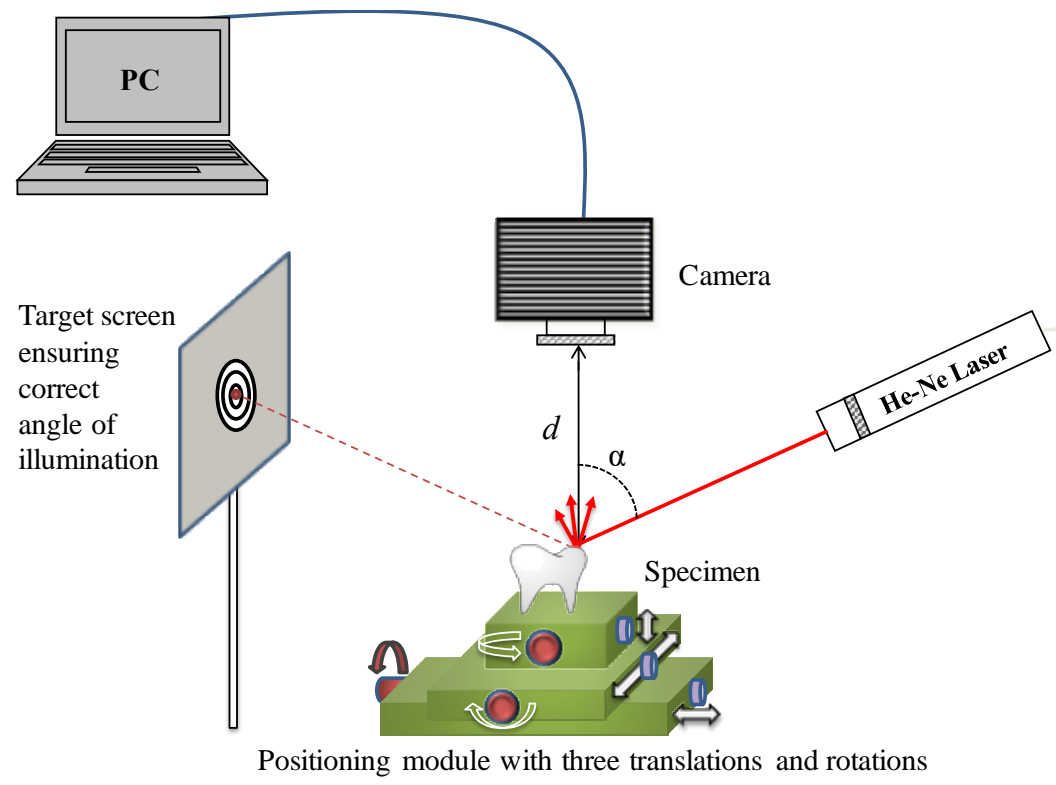

Fig. 1. (Color online) Optical set-up of the experiment.

The experimental set-up is based on the objective speckle pattern properties and does not require expensive camera lens system. 


\section{SPECIMEN PREPARATION}

For the purpose of our study a modern dental hybrid ceramic for CAD/CAM fabrication (Vita Enamic, Vita, Bad Sackingen, Germany) was used. Each of the specimens was polished with fine sandpaper with different grit sizes, producing surfaces with different roughness. Each surface received 500 strokes under a pressure of $10^{5} \mathrm{~Pa}$, in a fixed direction to ensure uniformity of the polishing process. A continuous water spray was applied during the process in order to remove any free particles. Two of the specimens (CM6 and CM7) received additional treatment. A polishing machine providing uniform polishing in all directions with sandpaper P5000 processed them. CM7 was additionally polished with a special polishing paste, to produce an even smoother surface. Description of the test subjects and their corresponding codes can be seen in Table 1.

Table 1. Codes for the test surfaces, as well as information about the polishing tools used to produce different levels of roughness. The grit sizes of the sandpaper are presented in the third row. CM6 and CM7 are the subjects that received extra polishing as mentioned above.

\begin{tabular}{ccccc}
\hline \hline $\begin{array}{c}\text { Specimen } \\
\text { code }\end{array}$ & $\begin{array}{c}\text { Polished by } \\
\text { sandpaper }\end{array}$ & $\begin{array}{c}\text { Number of } \\
\text { polishing strokes }\end{array}$ & $\begin{array}{c}\text { Sandpaper } \\
R q[\mu \mathrm{m}]\end{array}$ & $\begin{array}{c}\text { Specimen } \\
R q[\mu \mathrm{m}]\end{array}$ \\
\hline CM1 & P1000 & 500 & 10.23 & 2.2 \\
CM2 & P1200 & 500 & 7.89 & 1.16 \\
CM3 & P2000 & 500 & 5.22 & 0.93 \\
CM4 & P2500 & 500 & 3.81 & 0.80 \\
CM5 & P5000 & 500 & 2.71 & 0.66 \\
CM6 $^{*}$ & P5000 & Machine & 2.71 & 0.43 \\
CM7 $^{*}$ & P5000 & Machine + paste & 2.71 & 0.15 \\
\hline
\end{tabular}

Additionally, two dental crowns were fabricated using a commercial dental CAD/CAM milling unit Cerec MX (Sirona, Biberach, Germany). For this purpose, an acrylic maxillary right molar (Frasaco, Tettnang, Germany) was prepared according to the producers' recommendations. The prepared tooth was scanned and the crown is designed using an intraoral scanner (CerecBluecam, Sirona). The surface of the tested crown, used in this study is as followed:

- As milled: no surface treatment after milling;

- Polishing: For the polishing procedure a commercial dental polishing set, recommended by the producer, was used.

After the specimens were prepared, their surface roughness was examined by a commercial 3D optical profilometer Zeta-20. This examination was done to determine the Root-Mean-Square roughness (RMS) $-R q$ of the samples' surfaces simply 

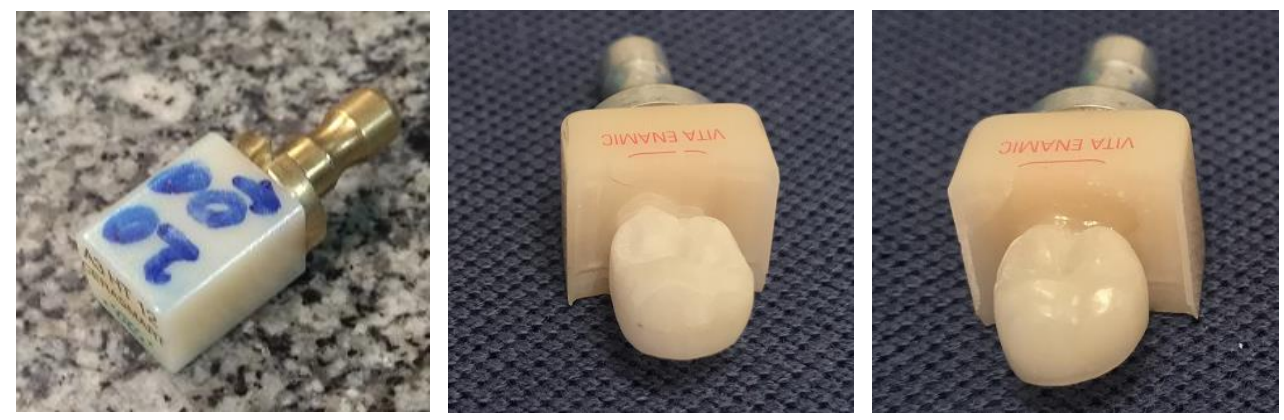

Fig. 2. (Color online) Example specimens - CM3 on the left, the unpolished crown in the middle, polished crown to the right.

as a reference. Each of the specimens was examined under the same magnification. Results for $R q$ may vary depending on the used magnification as estimation range goes from local to global. The surfaces were tested at various local areas and the presented RMS roughness is the result of averaging. Images of the observed specimens, captured by the optical profilometer, are presented on Fig. 3. An example of the specimens is presented in Fig. 2. RMS roughness value $R q$ for the unpolished crown was found to be $3.2 \mu \mathrm{m}$ and $0.28 \mu \mathrm{m}$ for the polished one.

\section{Contrast Estimation}

Speckle image contrast is a first-order statistics of the resultant speckle field. It depends on the surface roughness of diffusely reflecting objects. When estimating $V$ several factors influence the accuracy. Namely, these are the camera noise, inaccurate illumination angle, inaccurate distance between camera and surface, and specimen heating. In order to reduce the effects of the camera noise on $V$, multiple $M$ frames were taken and averaged. This is done in order to reduce additive camera noise. A clearer formulation of (1) can be expressed as

$$
V=\sqrt{\frac{\sum_{p=1}^{P} \sum_{q=1}^{Q}(\bar{I}(p, q)-\langle\bar{I}\rangle)^{2}}{(P Q-1)}} / \frac{\sum_{p=1}^{P} \sum_{q=1}^{Q} \bar{I}(p, q)}{P Q},
$$

where $P$ is number of columns, $Q$ is the number of rows, $P Q$ is the total number of pixels, and $\bar{I}$ is an estimate of the noise free intensity calculated by simple averaging of the consequently registered noisy images $I_{N_{i}}$

$$
\bar{I}=\frac{\sum_{i=1}^{M} I_{N_{i}}}{M} .
$$



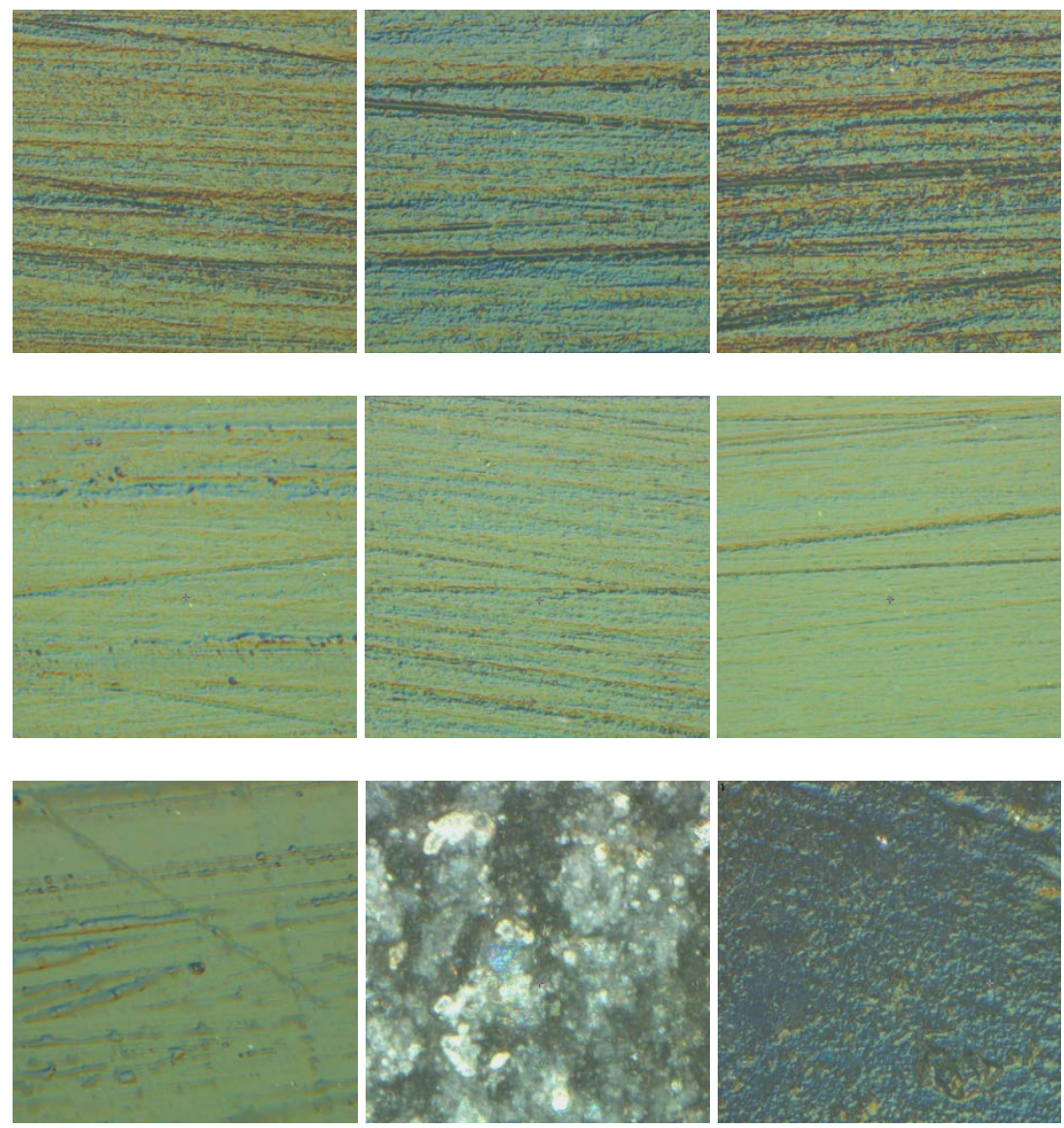

Fig. 3. (Color online) Zeta-20 images of the studied surfaces. First row, from left to right: CM1, CM2, CM3. Second row: CM4, CM5, CM6. Size of the examined surfaces is $141 \times$ $141 \mu \mathrm{m}$. Last row, from left to right: CM7, unpolished crown and polished crown.

Using formula (2) and (3), the speckle image contrast was calculated for the registered images.

Before data acquisition the dental crowns were thermally stabilized for several minutes to decrease the thermal roughness variations. Local examination of surface roughness was carried out using a non-expanded laser beam. This was done as the surfaces' shapes effect contrast estimation. A target screen is used to ensure the correct angle of incidence with respect to the examined surface. The screen is placed on a considerable distance from the scattering surface. The larger distance provides better control over the illumination angle set up. If needed an High-dynamic-range 
imaging (HDRI) [31-33] algorithm can be applied, which combines several frames with different exposures and produces an image with increased dynamic range.

\section{EXPERIMENTAL RESULTS}

Contrast estimation was carried out for an incidence angle $\alpha$ of $45^{\circ}$. A CMOS camera with 10 Mpix sensor matrix, 8 bit ADC, and $1.27 \times 1.27 \mu \mathrm{m}$ pixel size, capturing at $10 \mathrm{fps}$, was used to capture the images. A He-Ne laser with wavelength $\lambda$ of $633 \mathrm{~nm}$ with output power of $12 \mathrm{~mW}$ was used to illuminate the surface. The laser spot has a diameter of $1 \mathrm{~mm}$. Distance $d$ between camera and surface is $78.1 \mathrm{~mm}$.

Formula (2) was used to calculate $V$. All the specimens were placed under the laser beam for several minutes in order to reach thermal equilibrium. Results for the contrast are plotted against the results for the RMS roughness, obtained from the optical profilometer.

Measurement results show that speckle image contrast decreases with the increase of smoothness of the used polishing tools and the specimens themselves. The measurement uncertainty in calculating $V$ was evaluated at $4.8 \times 10^{-4}$ for $M=360$ frames. Errors of the same magnitude are introduced when a $1 \mathrm{~mm}$ change of distance between the pixel matrix and the surface is introduced. Additionally, a 0.03 degrees change in the illumination angle introduces an error of the same magnitude.

In order to demonstrate the effects of an object's transparency when measuring speckle image contrast, an evaluation for the sandpaper polishing tools was carried

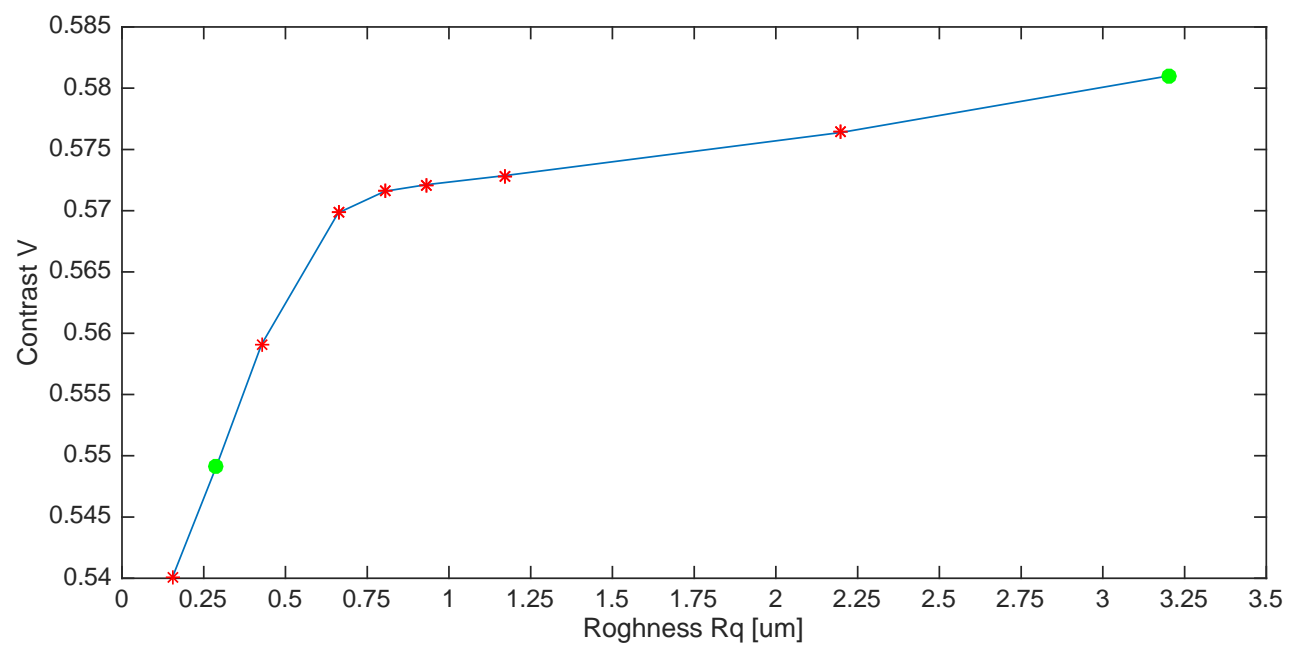

Fig. 4. (Color online) The contrast of the speckle image of the specimens. Marked with dots are the two dental crowns. The line, connecting the points, "leads the eye". 


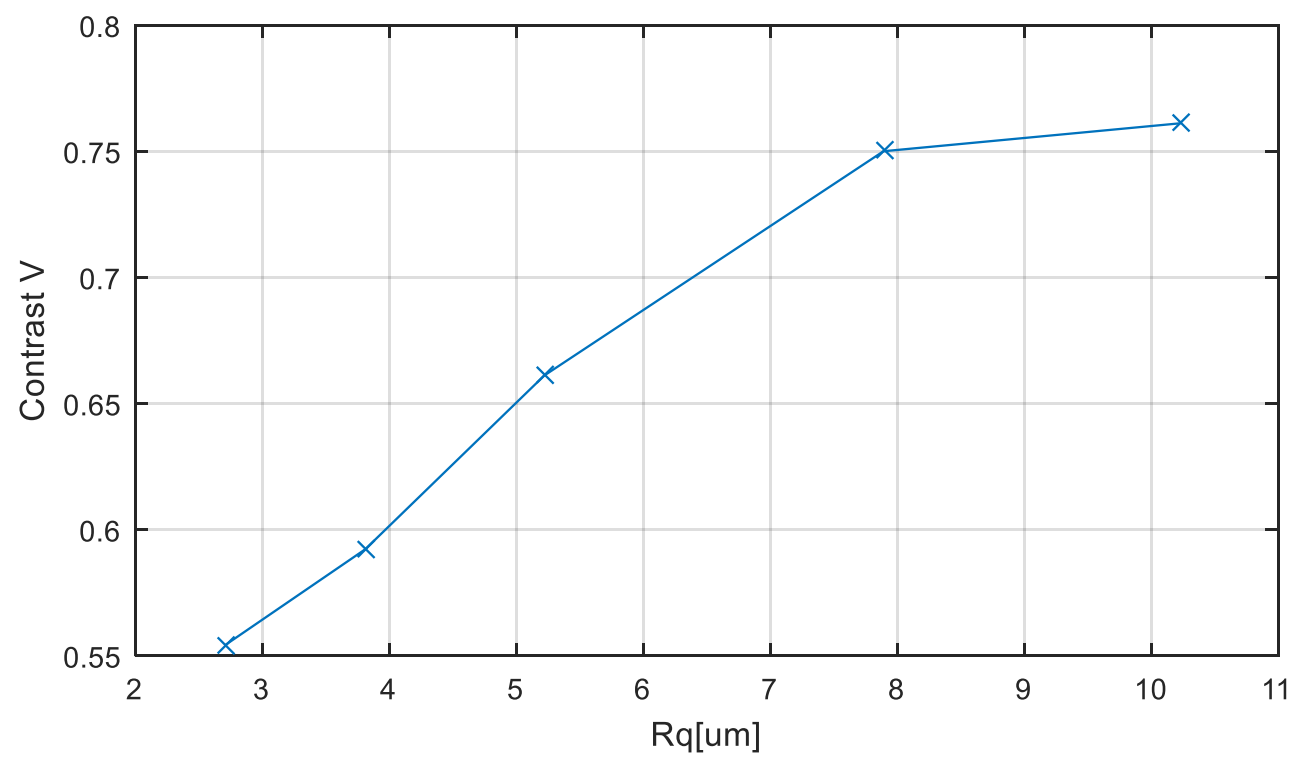

Fig. 5. (Color online) RMS roughness vs. Speckle contrast for the different sandpaper polishing surfaces. The line, connecting the points "leads the eye".

out as well. Experimental parameters such as $d$, illumination angle $\alpha$ and exposure were slightly different from the ones used above. As the RMS roughness of the sandpaper is greater and the specimens are not transparent, contrast estimation was done on only one frame. Results without averaging $(m=1)$ are again shown against the RMS roughness.

It was observed that the contrast varies stronger for non-transparent objects. For polished dental crowns variation in contrast ranges from 0.540 to 0.581 . For the sandpaper surfaces, the contrast variations are in the range of 0.552 to 0.761 . This substantial difference could be explained by the transparency of the crowns. For them some part of the recorded speckle pattern originates from the insides of the specimens. The sandpaper surfaces, however, are not transparent, and this significantly affects the range in which $V$ changes. As can be observed from Fig. 4 and Fig. 5 is that contrast estimation varies significantly for different types of surfaces and changes in the acquisition set-up. Thus, the estimations, produced by the technique can be regarded as relative, and should only be used when comparing objects of similar optical, structural and geometric properties. 


\section{Conclusion}

In this paper a method for surface roughness estimation of dental materials is proposed. A non-expanded laser beam was used for local examination of surface roughness. The dental crowns were thermally stabilized. Great emphasis was placed on ensuring illumination under the correct angle. The demonstrated technique is simple, reliable and does not require expensive optical systems and lenses. The method is based on a property of the resultant speckle pattern - namely the speckle image contrast. This property was calculated for different specimens prepared with sandpaper with various grit sizes. The behavior of $V$ to different roughness and different illumination angles was shown. This technique could be used for roughness estimation of dental crowns and ceramics in clinical environments. If a specimen with known root-mean-square roughness $R q$ has its speckle contrast evaluated, other specimens with unknown $R q$ can be compared to it after evaluation of their speckle contrast, thus providing a reliable way of collating them according to their smoothness.

\section{ACKNOWLEDGMENT}

The authors acknowledge the supported of the Bulgarian National Science Foundation grant B02/25 (2014) for the project "Clinical Wear Investigation of New Zirconia Dental Ceramics by Optical and Laser Techniques".

\section{REFERENCES}

[1] A. AL-WAHADNi, D. Martin (1999) An in vitro Investigation into the Wear Effects of Glazed, Unglazed and Refinished Dental Porcelain on an Opposing Material. Journal of Oral Rehabilitation 26 538-546.

[2] S. Heintze, A. Cavalleri, M. Forjanic, G. Zellweger, V. Rousson (2008) Wear of Ceramic and Antagonist - a Systematic Evaluation of Influencing Factors in vitro. Dental Materials 24 433-449.

[3] G. Mitov, S.D. Heintze, S. Walz, K. Woll, F. Muecklich, P. Pospiech (2012) Wear Behavior of Dental Y-TZP Ceramic against Natural Enamel after Different Finishing Procedures. Dental Materials 28 909-918.

[4] C. YilmaZ, T. KorkMAZ, H. DEMIR KÖPRÜLÚ, G. ERGÜN, Y. ÖZKAN (2008) Color Stability of Glazed and Polished Dental Porcelains. Journal of Prosthodontics 17 20-24.

[5] C.M. Bollenl, P. Lambrechts, M. Quirynen (1997) Comparison of Surface Roughness of Oral Hard Materials to the Threshold Surface Roughness for Bacterial Plaque Retention: A Review of the Literature. Dental Materials 13 258-269.

[6] K. Kawai, M. Urano, S. Ebisu (2000) Effect of Surface Roughness of Porcelain on Adhesion of Bacteria and Their Synthesizing Glucans. The Journal of Prosthetic Dentistry 83 664-667.

[7] J. Griggs, J. Thompson, K. Anusavice (1996) Effects of Flaw Size and AutoGlaze Treatment on Porcelain Strength. Journal of Dental Research 75 1414-1417. 
[8] N. De Jager, A. Feilzer, C. Davidson (2000) The Influence of Surface Roughness on Porcelain Strength. Dental Materials 16 381-388.

[9] H. FISCHER, M. SchäFER, R. MARX (2003) Effect of Surface Roughness on Flexural Strength of Veneer Ceramics. Journal of Dental Research 82 972-975.

[10] Y. Nakamura, S. Hojo, H. Sato (2010) The Effect of Surface Roughness on the Weibull Distribution of Porcelain Strength. Dental Materials Journal, 29 30-34.

[11] S. Flury, A. PeutzFeldt, A. Lussi (2012) Influence of Surface Roughness on Mechanical Properties of Two Computer-Aided Design/Computer-Aided Manufacturing (CAD/CAM) Ceramic Materials. Operative Dentistry 37 617-624.

[12] M. GhazAL, M. KERN (2009) The Influence of Antagonistic Surface Roughness on the Wear of Human Enamel and Nanofilled Composite Resin Artificial Teeth. The Journal of Prosthetic Dentistry 101 342-349.

[13] J. GARRATt (1982) A New Stylus Instrument with a Wide Dynamic Range for Use in Surface Metrology. Precision Engineering 4 145-151.

[14] G. Binnig, C.F. Quate, C. Gerber (1986) Atomic Force Microscope. Physical Review Letters 56 930-933.

[15] K.J. Stout, P.J. Sullivan, W.P. Dong, E. Mainsah, N. Luo, T. Mathia, H. ZahouAni (1993) "The Development of Methods for the Characterisation of Roughness in Three Dimensions". Commission of the European Communities, ISBN 18571 8023 2, Brussels-Luxembourg.

[16] J.C. WYANT (2002) White Light Interferometry. In: H.J. Caulfield (Ed.) "Holography: A Tribute to Yuri Denisyuk and Emmett Leith". Int. Society for Optics and Photonics, pp. 98-108.

[17] S. WANG, Y. TIAn, C.J. TAY (2003) Development of a Laser-Scattering-Based Probe for On-Line Measurement of Surface Roughness. Applied Optics 42 1318-1324.

[18] G.V. Blessing, J.A. Slotwinski, D.G. Eitzen, H.M. Ryan (1993) Ultrasonic Measurements of Surface Roughness. Applied Optics 32 3433-3437.

[19] D. Lockwood (2007) Light Scattering and Nanoscale Surface Roughness. In: A.A. Maradudin (Ed.) "Nanostructure Science and Technology". Springer, New York.

[20] A. Manallah, M. Bouafia (2011) Application of the Technique of Total Integrated Scattering of Light for Micro-Roughness Evaluation of Polished Surfaces. Physics Procedia 21 174-179.

[21] H. FuJiI, T. AsAKURA (1974) Effect of Surface Roughness on the Statistical Distribution of Image Speckle Intensity. Optics Communications 11 35-38.

[22] P. Lehmann (1999) Surface-Roughness Measurement Based on the Intensity Correlation Function of Scattered Light under Speckle-Pattern Illumination. Applied Optics 38 1144-1152.

[23] R.B. CRAne (1970) Use of a Laser-Produced Speckle Pattern to Determine Surface Roughness. Journal of the Optical Society of America 60 1658-1663.

[24] Y. Piederrière, J. Cariou, Y. Guern, B. Le Jeune, G. Le Brun, J. Lotrian (2004) Scattering through Fluids: Speckle Size Measurement and Monte Carlo Simulations Close to and into the Multiple Scattering. Optics Express 12 176-188. 
[25] A. Shulev, I. Roussev, S. Karpuzov, G. Stoilov, D. Ignatova, C. von See, G. Mitov (2016) Roughness Measurement of Dental Materials. Journal of Theoretical and Applied Mechanics, 46 27-36.

[26] D. LÉger, E. Mathieu, J. Perrin (1975) Optical Surface Roughness Determination Using Speckle Correlation Technique. Applied Optics 14 872-877.

[27] D. Youssef, H. El-Ghandoor, H. Kandel, J. El-Azab, S. Hassab-Elnaby (2017) Estimation of Articular Cartilage Surface Roughness Using Gray-Level CoOccurrence Matrix of Laser Speckle Image. Materials 10714.

[28] H.M. Pedersen (1976) Theory of Speckle Dependence on Surface Roughness. Journal of the Optical Society of America 66 1204-1210.

[29] J.W. Goodman (1975) Dependence of image Speckle Contrast on Surface Roughness. Optics Communications 14 324-327.

[30] N. TAKAi (1975) Relation between Statistical Properties of Surface Roughness and the Averaged Speckle Intensity in the Diffraction Field. Optics Communications 14 24-29.

[31] G. Cornuejols (1991) Image processing Device for Controlling the Transfer Function of an Optical System. In: Google Patents, https://patents.google.com/patent/EP0256051B1/en.

[32] O. Pirinen, A. Foi, A. Gotchev (2007) Color High Dynamic Range (HDR) Imaging in Luminance-Chrominance Space. In: Proceedings of the Third International Workshop on Video Processing and Quality Metrics for Consumer Electronics, Scottsdale, Arizona pp. 121-124.

[33] M. Goesele, W. Heidrich, B. Höfflinger, G. KrawczyK, K. Myszkowski, M. Trentacoste Less (2005) High Dynamic Range Techniques in Graphics: from Acquisition to Display. 10.2312/egt.20051056. 